\title{
Cartographic analysis of woodlice fauna of the former USSR
}

\author{
Daria M. Kuznetsova', Konstantin B. Gongalsky' \\ I A.N. Severtsov Institute of Ecology and Evolution, Russian Academy of Sciences, Moscow, Russia \\ Corresponding author: Konstantin B. Gongalsky (gongalsky@gmail.com)
}

Academic editor: S. Taiti | Received 15 November 2011 | Accepted 31 January 2012 | Published 20 March 2012

Citation: Kuznetsova DM, Gongalsky KB (2012) Cartographic analysis of woodlice fauna of the former USSR. In: Štrus J, Taiti S, Sfenthourakis S (Eds) Advances in Terrestrial Isopod Biology. ZooKeys 176: 1-11. doi: 10.3897/ zookeys.176.2372

\begin{abstract}
An inventory of the woodlice fauna of the former USSR yielded 190 species, 64 of them were recorded from the territory of Russia. According to the cartographic analysis, the limits of distribution of epigean terrestrial isopods over the area, excluding mountains, is explained by temperature. No woodlice records were found outside the isocline of 120 days a year with the mean daily air temperature $>10^{\circ} \mathrm{C}$. The highest species diversity was found between the isoclines of 180 and 210 days. These areas correspond to forest-steppe and steppe zones.
\end{abstract}

\section{Keywords}

Woodlice, mean annual air temperature, database, Russia

\section{Introduction}

Studies of spatial differentiation of various taxa are among the most important frontiers of modern biogeography. For some well-studied groups, mainly, vertebrates and plants, such trends are already discovered (Loiselle et al. 2003; Guisan and Thuiller 2005; Grenouillet et al. 2011), but for soil-dwelling invertebrates they are only at the stage of species inventory. However, there are certain groups of invertebrates for which analysis of spatial differentiation is already possible due to the large number of records from different geographical localities. Woodlice are among such groups.

There is no faunistic list of terrestrial isopods for the territory of the former USSR until now, as well as of the territory of Russia. However, there are extensive regional lists (Borutzky 1948, 1953; Zalesskaya and Rybalov 1982; Khisametdinova 2007;

Copyright D.M. Kuznetsova, K.B. Gongalsky. This is an open access article distributed under the terms of the Creative Commons Attribution License 3.0 (CC-BY), which permits unrestricted use, distribution, and reproduction in any medium, provided the original author and source are credited. 
Gongalsky and Kuznetsova 2011), and numerous records scattered in the literature devoted to soil macrofauna. At the same time, there are only a few ecological studies about factors affecting woodlice distribution over regions of the former USSR (Gongalsky et al. 2005; Khisametdinova 2009).

The aim of the study is to determine the factors affecting woodlice distribution over the plain area of the former Soviet Union. To achieve this, an inventory of species distribution across the study area was made. The task was to create a database indicating locations with woodlice presence/absence overlaid with several environmental variable values distribution.

\section{Material and methods}

\section{Database}

The first step was to compile a list of species for the study area. We made a database of isopod presence or absence in the locations across the whole territory of the former USSR (both plains and mountains). For each record the database includes information about date, data source, geographical coordinates, location, isopod species list or information about woodlice absence in the soil fauna list, biotope, and natural zone.

Three types of information sources of terrestrial isopod locations were used: i) available literature on soil fauna surveys; ii) collections of the Zoological Museum of Moscow State University (Moscow, Russia) and the Zoological Institute of the Russian Academy of Sciences (St.-Petersburg, Russia); and iii) authors' personal collections. Here we provide a list of woodlice from the territory of the former USSR since some species and localities were not included in the list of Schmalfuss (2003), although it covered the majority of species. To work with regional databases, a specific list would be useful. Since such a list for this area did not exist, the proposed compilation would be a start to be completed in the future. We used the taxonomic system proposed by Schmalfuss (2003) for species naming. Isopod absence was recorded only in extensively surveyed locations.

For cartographic analysis, 259 locations were chosen, 44 of which with woodlice absence. Due to the difficulty of tracing ecological trends in the mountains, only plain territories were involved into the analysis. Some species were excluded from the analysis: i) synanthropic species and ii) species inhabiting azonal locations, such as sea coasts, caves and anthills.

Then database records with isopod presence or absence locations were laid on the geographic maps to perform cartographic analysis.

\section{Cartographic analysis}

The map of woodlice distribution was visually compared with the maps of environmental factors (mean annual temperature; the period with temperature above $10^{\circ} \mathrm{C}$; 
mean precipitation; permafrost distribution; soil $\mathrm{pH}$ and soil type; vegetation type; natural zones) found in the Agricultural Atlas of the USSR (Tulupnikov 1960) and the Geographical Atlas of the USSR (Kolosova 1980). The data were verified using the WorldClim database (Hijmans et al. 2005).

The database is maintained in MS Excel. Cartographic analysis is done in MapInfo 8.5.

\section{Results and discussion}

\section{Limits of isopod distribution}

Woodlice have not been recorded northwards the isocline of 120 days a year with temperature $>10^{\circ} \mathrm{C}$ (Fig. 1). The northern border of woodlice distribution matches the distribution of this parameter. Other parameters did not coincide with isopod distribution as well as with this isocline (data not shown).

\section{Species diversity}

In total, 190 species were recorded from the territory of the former USSR (Appendix 1). Among them, 64 were recorded from the territory of Russia. Northernmost natural

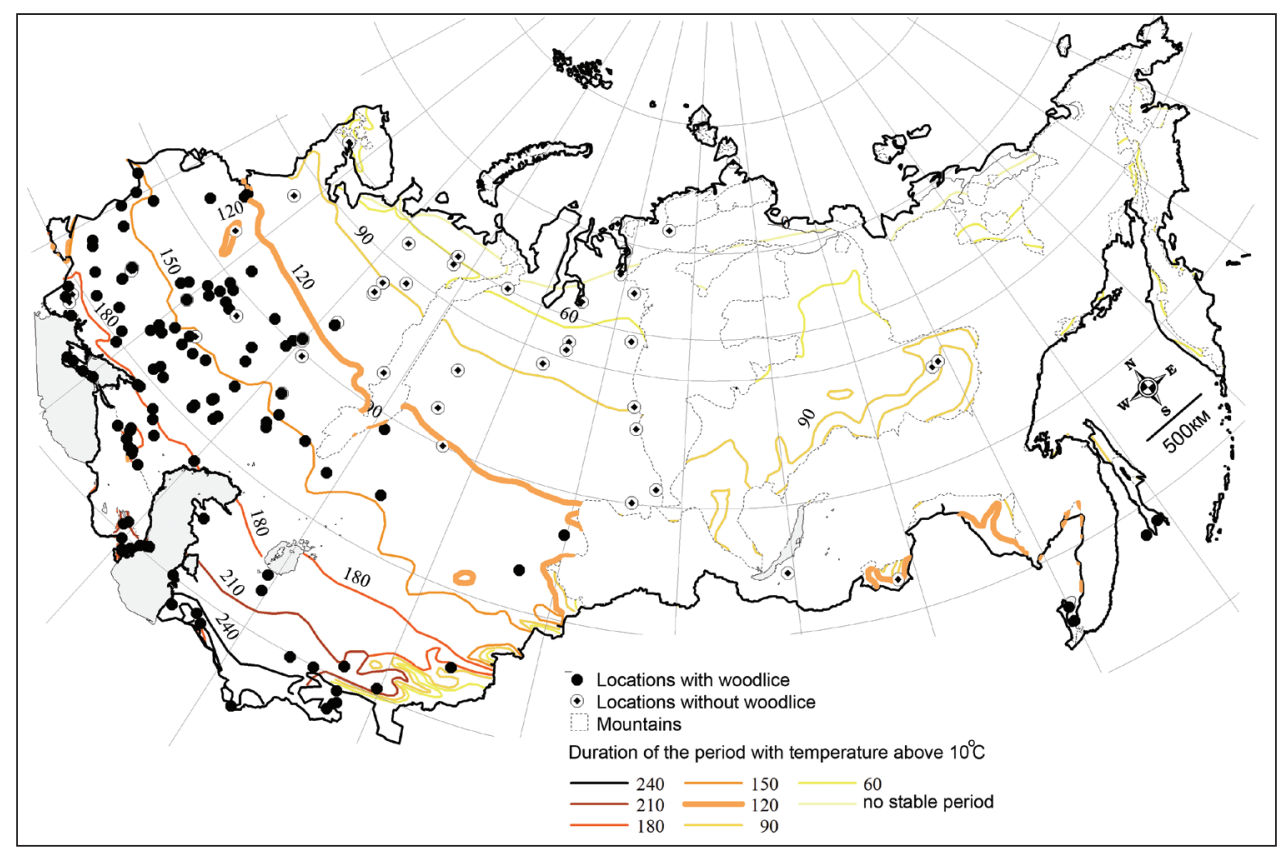

Figure I. Map of woodlice presence or absence over the plain territory of the former USSR. The duration of period with temperature $>10^{\circ} \mathrm{C}$ is adapted from Geographical Atlas of the USSR (Kolosova 1980). 
zone with woodlice records is southern taiga. No woodlice records were in tundra, northern and middle taiga. The species diversity increases southwards, but decreases in the deserts. However, this may be due to the low number of locations extensively studied to reveal local faunas.

Distribution of isopods is known to be limited by natural factors, such as temperature and moisture (Harding and Sutton 1985, Hopkin 1991). In our study, the limiting factor of woodlice distribution towards the north turned out to be the length of the warm period, expressed as number of days when the temperature was above $10^{\circ} \mathrm{C}$. The highest species diversity was observed between isoclines of 180 and 210 days with temperature $>10^{\circ} \mathrm{C}$. Colder conditions slow down their physiological processes (Hopkin 1991) and limit their distribution. For a better understanding of distribution of woodlice, a Species Distribution Modeling (Elith and Leathwick 2009, Franklin 2009) should be applied, which is a next step in the analysis of the database of Russian isopods.

\section{Acknowledgements}

The authors are grateful to Dr H. Schmalfuss and Dr Ch. Schmidt for the help with isopod identifications, and to Dr K.G. Mikhailov and Dr B.V. Mezhov for allowing working with crustacean material kept at Zoological Museum of Moscow State University.

The study is supported by Russian Foundation for Basic Research (grant 11-0400245) and the Program "Biodiversity" of the Presidium of the Russian Academy of Sciences.

\section{References}

Borutzky EV (1948) To the fauna of spring Isopoda of Middle Asia. Sbornik Trudov zoologicheskogo Muzeya, 274-279. [in Russian]

Borutzky EV (1953) To the fauna of woodlice of Tadjikistan. Izvestiya Akademii Nauk Tadjikskoi SSR 3: 15-22. [in Russian]

Elith J, Leathwick JR (2009) Species distribution models: Ecological explanation and prediction across space and time. Annual Review of Ecology, Evolution, and Systematics 40: 677-697. doi: 10.1146/annurev.ecolsys.110308.120159

Franklin J (2009) Mapping species distributions: Spatial inference and prediction. Cambridge University Press, Cambridge, 338 pp.

Gongalsky KB, Kuznetsova DM (2011) Fauna and population of woodlice (Isopoda, Oniscidea) of Abrau Peninsula, North-West Caucasus. Zoologicheskii Zhurnal 90: 916-922. [in Russian]

Gongalsky KB, Savin FA, Pokarzhevskii AD, Filimonova ZV (2005) Spatial distribution of isopods in an oak-beech forest. European Journal of Soil Biology 41: 117-122. doi: 10.1016/j.ejsobi.2005.09.012 
Grenouillet G, Buisson L, Casajus N, Lek S (2011) Ensemble modelling of species distribution: the effects of geographical and environmental ranges. Ecography 34: 9-17. doi: 10.1111/j.1600-0587.2010.06152.x

Guisan A, Thuiller W (2005) Predicting species distribution: offering more than simple habitat models. Ecology Letters 8: 993-1009. doi: 10.1111/j.1461-0248.2005.00792.x

Harding PT, Sutton SL (1985) Woodlice in Britain and Ireland; Distribution and habitat. Institute of Terrestial Ecology, Huntingdon, $152 \mathrm{pp}$.

Hijmans RJ, Cameron SE, Parra JL, Jones PG, Jarvis A (2005) Very high resolution interpolated climate surfaces for global land areas. International Journal of Climatology 25: 1965-1978. doi: 10.1002/joc.1276

Hopkin S (1991) A key to the woodlice of Britain and Ireland. An AIDGAP key. Richmond Publishing, Slough.

Khisametdinova DD (2007) To the fauna of woodlice (Isopoda, Oniscidea) of Rostov Region. Izvestia vuzov. Severo-Kavkazskiy Region. Yestestvennye Nauki 6: 86-87. [in Russian]

Khisametdinova DD (2009) Ecologic-faunistic characteristic of woodlice (Isopoda, Oniscidea) of the Lower Don River. PhD Thesis. Southern Federal University, Rostov-on-Don. [in Russian]

Kolosova LN (Ed) (1980) Geographical Atlas of the USSR for schoolteachers. Vysshaya Shkola, Moscow, 238 pp. [in Russian]

Loiselle BA, Howell CA, Graham CH, Goerck JM, Brooks T, Smith KG, Williams PH (2003) Avoiding pitfalls of using species distribution models in conservation planning. Conservation Biology 17: 1591-1600. doi: 10.1111/j.1523-1739.2003.00233.x

Schmalfuss H (2003) World catalog of terrestrial isopods (Isopoda: Oniscidea). Stuttgarter Beiträge zur Naturkunde, Serie A, Nr. 654, 341 pp.

Schmalfuss H, Wolf-Schwenninger K (2002) A bibliography of terrestrial isopods (Crustacea, Isopoda, Oniscidea). Stuttgarter Beitrage zur Naturkunde, Serie A, Nr. 639, 120 pp.

Tulupnikov AI (Ed) (1960) Agricultural Atlas of the USSR. GUGK, Moscow, 308 pp. [in Russian]

Zalesskaya NT, Rybalov LV (1982) Fauna of woodlice of Moscow Region (Crustacea, Isopoda, Oniscidea). In: Gilyarov MS (Ed.) Soil invertebrates of Moscow Region. Nauka Publ., Moscow, 171-179. [in Russian] 


\section{Appendix I}

List of woodlice species from the territory of the former USSR. Abbreviations: Ab - Abkhazia, Ar - Armenia, Az - Azerbaijan, Bl - Belarus, Ge - Georgia, Kz - Kazakhstan, Kg - Kyrgyzstan, Lt - Lithuania, Md - Moldova, Ru - Russia, Td - Tajikistan, Tu - Turkmenistan, Ua - Ukraine, Uz - Uzbekistan; S, N, W, E - south, north, west, east. References to authorships of the species can be found in Schmalfuss and Wolf-Schwenninger (2002).

\begin{tabular}{|c|c|c|}
\hline 1 & Acaeroplastes kosswigi Verhoeff, 1941 & Az: Nabran’ \\
\hline 2 & Agabiformius orientalis (Dollfus, 1905) & Ru: Volgograd region \\
\hline 3 & $\begin{array}{l}\text { Armadillidium azerbaidzhanum Schmalfuss, } \\
1990\end{array}$ & $\begin{array}{l}\text { Ar: Khastarak; Az: Baku, Adjikend, Drmbon, } \\
\text { Lenkoran, Salyany, Sheki; Ge: Kakhetia, } \\
\text { Vashlovan; Ru: Rostov, Stavropol regions, N } \\
\text { Osetia }\end{array}$ \\
\hline 4 & Armadillidium granulatum Brandt, 1833 & Ru: Krasnodar region; Ua: S Crimea \\
\hline 5 & Armadillidium nasatum Budde-Lund, 1885 & Ru: Moscow; Ab: Sukhum \\
\hline 6 & Armadillidium opacum (C.Koch, 1841) & Ua: Kiev \\
\hline 7 & Armadillidium pallasii Brandt, 1833 & $\begin{array}{l}\text { Ab: Sukhum; Ua: Crimea, Odessa; Ru: Krasnodar } \\
\text { region }\end{array}$ \\
\hline 8 & Armadillidium pictum Brandt, 1833 & Ua: Crimea \\
\hline 9 & Armadillidium pulchellum (Zenker, 1798) & Lt: Vilnius \\
\hline 10 & Armadillidium traiani Demianowicz, 1932 & $\mathrm{Md}$ \\
\hline 11 & Armadillidium versicolor Stein, 1859 & Ru: Penza, Saratov, Tula regions \\
\hline 12 & Armadillidium vulgare Latreille, 1804 & $\begin{array}{l}\text { Ab: Sukhum; Az; Ge: Adygeni, Tbilisi; Ru: } \\
\text { Dagestan, Krasnodar, Kaluga, Volgograd, Rostov } \\
\text { regions; Ua: Crimea, Kiev, Odessa }\end{array}$ \\
\hline 13 & Armadillidium zenckeri Brandt, 1833 & Ua: Crimea, Zakaspyisk region \\
\hline 14 & Armadillo alievi Schmalfuss, 1990 & Az: Baku, Kobustan \\
\hline 15 & Armadillo officinalis Dumeril, 1816 & Ru: Krasnodar region; Ua: S Crimea, Odessa \\
\hline 16 & Armadilloniscus ellipticus (Harger, 1878) & Ru: Krasnodar region; Ab: Gagry \\
\hline 17 & Borutzkyella revasi (Borutzky, 1973) & Ab: Gudauty region \\
\hline 18 & Buddelundiella cataractae Verhoeff, 1930 & Ge: Tskhaltubo \\
\hline 19 & Caucasocyphonethes cavaticus Borutzky, 1948 & Ru: Krasnodar region \\
\hline 20 & Caucasoligidium cavernicola Borutzky, 1950 & Ab: Gudauty, Sukhum; Ge: Gogolety \\
\hline 21 & Caucasonethes borutzkyi Verhoeff, 1932 & Ge: Tskhaltubo \\
\hline 22 & Chaetophiloscia cellaria Dollfus, 1884 & Ru: Rostov region \\
\hline 23 & Chaetophiloscia elongata Dollfus, 1884 & Ua: Crimea \\
\hline 24 & Chaetophiloscia hastata Verhoeff, 1929 & Ru: Krasnodar region \\
\hline 25 & Colchidoniscus kutaissianus Borutzky, 1974 & Ge: Tskhaltubo \\
\hline 26 & Cylisticoides angulatus Schmalfuss, 2003 & $\begin{array}{l}\text { Az: Istisu, Lenkoran, Yardymly; Kz: Astana; Ru: } \\
\text { Krasnodar region }\end{array}$ \\
\hline 27 & Cylisticus albomaculatus Borutzky, 1957 & Ru: Rostov, Voronezh, Volgograd regions \\
\hline 28 & Cylisticus arnoldii Borutzky, 1961 & Ua: Kharkov, Zmiev \\
\hline 29 & Cylisticus birsteini Borutzky, 1961 & Ru: Krasnodar region \\
\hline 30 & Cylisticus caucasius Verhoeff, 1917 & $\begin{array}{l}\text { Ab: Gudauty, Gagry, Kelassuri, Sukhum; } \\
\text { Ge: Kutaisi, Tkibuli, Tskhaltubo, Shovi; Ru: } \\
\text { Krasnodar region }\end{array}$ \\
\hline
\end{tabular}




\begin{tabular}{|c|c|c|}
\hline 31 & Cylisticus convexus De Geer, 1778 & $\begin{array}{l}\text { Ru: Chelyabinsk, Kaluga, Moscow, Rostov, } \\
\text { Voronezh regions, Ua: S Crimea, Kiev }\end{array}$ \\
\hline 32 & Cylisticus cretaceus Borutzky, 1957 & Ru: Rostov region; Ua: Lugansk region \\
\hline 33 & Cylisticus desertorum Borutzky, 1957 & Ru: Rostov, Stavropol regions; Ua \\
\hline 34 & Cylisticus giljarovi Borutzky, 1977 & $\begin{array}{l}\text { Ru: Adygea, Stavropol, Krasnodar regions, N } \\
\text { Osetia }\end{array}$ \\
\hline 35 & Cylisticus iners Budde-Lund, 1880 & $\begin{array}{l}\text { Ar: Azizbekov, Leninakan, Tshakhkavan; Az: } \\
\text { Airidja, Mardakert, Zakatalinsk regions; Ru: } \\
\text { Chechnya; Ge: Manglisi, Shuahevi }\end{array}$ \\
\hline 36 & Cylisticus lencoranensis Borutzky, 1977 & Az: Prishib \\
\hline 37 & Cylisticus mitis Budde-Lund, 1885 & Ge: Kutaisi \\
\hline 38 & Cylisticus orientalis Borutzky, 1939 & Ru: Orenburg region \\
\hline 39 & Cylisticus rotabilis Budde-Lund, 1885 & Ua: S Crimea \\
\hline 40 & Cylisticus sarmaticus Borutzky, 1977 & Ru: Rostov region; Ua: Zaporozhye region \\
\hline 41 & Cylisticus silvestris Borutzky, 1957 & Ru: Moscow, Rostov, Stavropol regions \\
\hline 42 & Cylisticus strouhali Borutzky, 1977 & Ar: W Vanadzor, Spitaki \\
\hline 43 & Desertoniscus birsteini Borutzky, 1945 & $\mathrm{Tu}$ \\
\hline 44 & Desertoniscus bulbifrons Borutzky, 1945 & $\mathrm{Tu}$ \\
\hline 45 & Desertoniscus elongatus Borutzky, 1945 & $\mathrm{Tu}$ \\
\hline 46 & Desertoniscus kirghizicus Borutzky, 1978 & $\mathrm{Kg}$ \\
\hline 47 & Desertoniscus reductus Borutzky, 1978 & $\mathrm{Td}$ \\
\hline 48 & Desertoniscus subterraneus Verhoeff, 1930 & Kg; Tu: (Kizil-arvat); Td: Samgar massif \\
\hline 49 & Desertoniscus tekinus Borutzky, 1945 & $\mathrm{Tu}$ \\
\hline 50 & Desertoniscus zhelochovtzevi Borutzky, 1945 & $\mathrm{Uz}$ \\
\hline 51 & Detonella papillicornis (Richardson, 1904) & Ru: Kamchatka, Sakhalin regions \\
\hline 52 & Halophiloscia couchii (Kinahan, 1858) & Ru: Krasnodar region; Ua: Crimea \\
\hline 53 & Haplophthalmus danicus Budde-Lund, 1880 & Ru: Krasnodar, Rostov regions \\
\hline 54 & Hemilepistoides messerianus Borutzky, 1945 & $\mathrm{Tu}$ \\
\hline 55 & Hemilepistus buddelundi Borutzky, 1945 & $\mathrm{Tu}$ \\
\hline 56 & Hemilepistus communis Borutzky, 1945 & Td: Samgar massif \\
\hline 57 & Hemilepistus crenulatus (Pallas, 1771) & $\begin{array}{l}\text { Td: Samgar massif; Tu: Central Karakum, Kyzyl- } \\
\text { Arvat; Uz: Zakaspiysk, Fergana regions; Kg }\end{array}$ \\
\hline 58 & Hemilepistus cristatus Budde-Lund, 1885 & Tu: Kyzyl-Arvat \\
\hline 59 & Hemilepistus elongatus Budde-Lund, 1885 & Ru: Rostov, Stavropol regions; Tu: SW part \\
\hline 60 & Hemilepistus fedtschenkoi (Uljanin, 1875) & $\begin{array}{l}\text { Kz: Semipalatinsk; Tu: Krasnovodsk; Uz: Bukhara, } \\
\text { Samarkand, Syrdaryinsk, Zakaspyisk regions }\end{array}$ \\
\hline 61 & Hemilepistus heptneri Borutzky, 1945 & $\mathrm{Tu}$ \\
\hline 62 & Hemilepistus klugii (Brandt, 1833) & Az: Baku \\
\hline 63 & Hemilepistus magnus Borutzky, 1945 & $\mathrm{Uz}_{\mathrm{z}}$ \\
\hline 64 & Hemilepistus nodosus Budde-Lund, 1885 & $\mathrm{Tu} ; \mathrm{Kz}$ \\
\hline 65 & Hemilepistus pavlovskii Borutzky, 1954 & $\mathrm{Kz}$ \\
\hline 66 & Hemilepistus reductus Borutzky, 1945 & Uz: Bukhara, Samarkand, Syrdaryinsk regions \\
\hline 67 & Hemilepistus rhinoceros Borutzky, 1958 & $\mathrm{Kz}$ \\
\hline 68 & Hemilepistus ruderalis (Pallas, 1771) & Ru: Volgograd region; Kz: Djanybek \\
\hline 69 & Hemilepistus russonovae Borutzky, 1951 & Az: Baku \\
\hline 70 & Hemilepistus zachvatkini Verhoeff, 1930 & Td: Samgar massif \\
\hline
\end{tabular}




\begin{tabular}{|c|c|c|}
\hline 71 & Hyloniscus riparius C. Koch, 1838 & $\begin{array}{l}\text { Ru: Moscow, Penza, Pskov, Rostov, Tula regions; } \\
\text { Ua: Kiev region }\end{array}$ \\
\hline 72 & Leptotrichus panzerii (Audonin, 1826) & Ua: Crimea \\
\hline 73 & Leptotrichus tauricus Budde-Lund, 1885 & Ua: Crimea \\
\hline 74 & Ligia cinerascens Budde-Lund, 1885 & Ru: Kurily islands \\
\hline 75 & Ligia italica Fabricius, 1798 & Ua: Crimea \\
\hline 76 & Ligia pallasii Brandt, 1833 & Kadakh? \\
\hline 77 & Ligidium birsteini Borutzky, 1950 & Ab: Gagry \\
\hline 78 & Ligidium cavaticum Borutzky, 1950 & Ru: Krasnodar region \\
\hline 79 & Ligidium fragile Budde-Lund, 1885 & Ab: Sukhum \\
\hline 80 & Ligidium germanicum Verhoeff, 1901 & Md \\
\hline 81 & Ligidium hypnorum Cuvier, 1792 & $\begin{array}{l}\text { Ab: Sukhum; Bl: Belovezha National Park; Ru: } \\
\text { Tver, Kaluga, Moscow regions; Ua: Crimea, Kiev }\end{array}$ \\
\hline 82 & Ligidium margaritae Borutzky, 1955 & Kz: Alma-Ata \\
\hline 83 & Ligidium nodulosum Verhoeff, 1918 & Ab: Gagry \\
\hline 84 & Ligidium shadini Borutzky, 1948 & $\mathrm{Td}$ \\
\hline 85 & Ligidium tauricum Verhoeff, 1930 & Ua: Crimea \\
\hline 86 & Ligidium zaitzevi Borutzky, 1950 & Ab: Sukhum \\
\hline 87 & Ligidium zernovi Borutzky, 1948 & $\mathrm{Kg}$ \\
\hline 88 & Mingrelloniscus inchhuricus Borutzky, 1974 & Ge: Megrelia \\
\hline 89 & Nagurus matekini Borutzky, 1959 & $\mathrm{Kg}$ \\
\hline 90 & Oniscus asellus Linne, 1758 & Lt: Vilnius; Ru: Pskov region; Ua: Kiev \\
\hline 91 & Parcylisticus armenicus Borutzky, 1970 & Ar: Daralagez \\
\hline 92 & Parcylisticus dentifrons Budde-Lund, 1885 & $\begin{array}{l}\text { Az: Kutkashen; Ge: Manglisi; Ru: Astrakhan, } \\
\text { Stavropol regions, Chechnya, Dagestan, } \\
\text { Kabardino-Balkaria, N Osetia; Ua: Crimea }\end{array}$ \\
\hline 93 & Parcylisticus georgianus Schmalfuss, 2003 & Ge: Adigeni, Batumi, Kutaisi, Mestia \\
\hline 94 & Parcylisticus golovatchi Schmalfuss, 2003 & Az: Shikahokh \\
\hline 95 & Parcylisticus mrovdaghicus (Borutzky, 1970) & $\begin{array}{l}\text { Az: Avash, Dashsalty, Kelbadjar, Kirovobad, } \\
\text { Lenkoran, Zuvand }\end{array}$ \\
\hline 96 & Parcylisticus urartuensis Borutzky, 1970 & $\mathrm{Ar}$ \\
\hline 97 & Parcylisticus zangezuricus Borutzky, 1970 & $\mathrm{Ar}$ \\
\hline 98 & Platyarthrus armenicus Borutzky, 1976 & Ar: Megri \\
\hline 99 & Platyarthrus hoffmannseggii Brandt, 1833 & Ru: Krasnodar region \\
\hline 100 & Platyarthrus luppovae Borutzky, 1953 & $\mathrm{Td}$ \\
\hline 101 & Platyarthrus mesasiaticus Borutzky, 1976 & $\mathrm{Tu}$ \\
\hline 102 & Platyarthrus ocellatus Borutzky, 1953 & $\mathrm{Td}$ \\
\hline 103 & Platyarthrus schoblii Budde-Lund, 1885 & Ua: Crimea \\
\hline 104 & Porcellio bistriatus Budde-Lund, 1885 & Ab: Sukhum; Ru: Krasnodar region \\
\hline 105 & Porcellio crassicornis C. Koch, 1841 & Bl: Minsk \\
\hline 106 & Porcellio dilatatus Brandt, 1833 & Ar: Sevan \\
\hline 107 & Porcellio laevis Latreille, 1804 & $\begin{array}{l}\text { Ab: Sukhum; Ru: Altay, Kalmykia, Moscow, } \\
\text { Primorie, Rostov regions; Ua: S Crimea, Odessa; } \\
\text { Uz: Bukhara region }\end{array}$ \\
\hline 108 & Porcellio lamellatus Budde-Lund, 1885 & Ua: Crimea \\
\hline 109 & Porcellio obsoletus Budde-Lund, 1885 & Ua: S Crimea \\
\hline
\end{tabular}




\begin{tabular}{|c|c|c|}
\hline 110 & Porcellio scaber Latreille, 1804 & $\begin{array}{l}\text { Bl: Belovezha, Berezinsky reserves; Lt: Vilnius; Ru: } \\
\text { Belgorod, Kaluga, Moscow, Nizhni Novgorod, } \\
\text { Primorie, Rostov regions, Kamchatka, S } \\
\text { Kuruly islands, Sakhalin; Ua: Kiev, Kremenetz, } \\
\text { Vinnickaya region (Yampol') }\end{array}$ \\
\hline 111 & Porcellio spinicornis Say, 1818 & $\begin{array}{l}\text { Bl: Minsk; Lt: Vilnius; Md; Ru: Kaluga, } \\
\text { Leningrad, Moscow Pskov regions; Ua: Kiev }\end{array}$ \\
\hline 112 & Porcellio uljanini Budde-Lund, 1885 & Ua: Crimea \\
\hline 113 & Porcellio variabilis Lucas, 1849 & Ua: Crimea \\
\hline 114 & $\begin{array}{l}\text { Porcellionides approximatus Budde-Lund, } \\
1885\end{array}$ & Md; Ru: Stavropol region; Ua: Crimea \\
\hline 115 & Porcellionides linearis (Budde-Lund, 1885) & Uz: Nukus \\
\hline 116 & Porcellionides pruinosus Brandt, 1833 & $\begin{array}{l}\text { Ab: Sukhum; Ar: Shorzha; Az: Baku, Khachmas, } \\
\text { Nabran; Ru: Baikal, Volgograd, Krasnodar region, } \\
\text { Moscow, Rostov, Saratov regions; Ua: Crimea }\end{array}$ \\
\hline 117 & Porcellionides rectifrons (Budde-Lund, 1885) & Ua: Crimea \\
\hline 118 & Porcellium collicola (Verhoeff, 1907) & $\mathrm{Md}$ \\
\hline 119 & Porcellium conspersum C. Koch, 1841 & Bl: Belovezha Reserve; Ua \\
\hline 120 & Protracheoniscus abricossovi Borutzky, 1945 & $\mathrm{Tu}$ \\
\hline 121 & Protracheoniscus alabashensis Borutzky, 1959 & $\mathrm{Kg}$ \\
\hline 122 & Protracheoniscus almaatinus Borutzky, 1975 & Kz: Alma-Ata \\
\hline 123 & Protracheoniscus anatolii Borutzky, 1959 & $\mathrm{Kg}$ \\
\hline 124 & Protracheoniscus armenicus Borutzky, 1975 & Ge: Megri \\
\hline 125 & Protracheoniscus asiaticus (Uljanin, 1875) & $\begin{array}{l}\text { Ru: Moscow, Nizhni Novgorod, Rostov, Ryazan' } \\
\text { regions; Td: Smagar massif }\end{array}$ \\
\hline 126 & Protracheoniscus atrecicus Borutzky, 1945 & Tu: Bugdaily \\
\hline 127 & $\begin{array}{l}\text { Protracheoniscus bugdajliensis Borutzky, } \\
1975\end{array}$ & Tu: Bugdaily \\
\hline 128 & Protracheoniscus cristatus Borutzky, 1945 & Az: Lenkoran, Sara isl.; Tu \\
\hline 129 & Protracheoniscus darevskii Borutzky, 1975 & Ar: Megri \\
\hline 130 & Protracheoniscus delilensis Borutzky, 1945 & $\mathrm{Tu}$ \\
\hline 131 & Protracheoniscus desertorum Verhoeff, 1930 & Turkestan? \\
\hline 132 & Protracheoniscus digitifer Borutzky, 1945 & $\mathrm{Tu}$ \\
\hline 133 & Protracheoniscus fossuliger Verhoff, 1901 & Ru: Krasnodar, Rostov regions \\
\hline 134 & Protracheoniscus giljarovi Borutzky, 1957 & Ru: Rostov region; Ua: Lugansk region \\
\hline 135 & Protracheoniscus gissarensis Borutzky, 1975 & Td: Dushanbe \\
\hline 136 & Protracheoniscus hirsutulus Verhoff, 1930 & Uz: Tashkent \\
\hline 137 & Protracheoniscus kopetdagicus Borutzky, 1945 & $\mathrm{Tu}$ \\
\hline 138 & $\begin{array}{l}\text { Protracheoniscus kryszanovskii Borutzky, } \\
1957\end{array}$ & Ru: Volgograd region, Kalmykia \\
\hline 139 & Protracheoniscus latus (Uljanin, 1875) & Td: Zeravshvan valley \\
\hline 140 & $\begin{array}{l}\text { Protracheoniscus litoralis (Budde-Lund, } \\
\text { 1885) }\end{array}$ & Ua: Crimea \\
\hline 141 & Protracheoniscus major (Dollfus, 1903) & Ru: Rostov region; Ua: Kiev \\
\hline 142 & $\begin{array}{l}\text { Protracheoniscus maracandicus (Uljanin, } \\
1875 \text { ) }\end{array}$ & $\begin{array}{l}\text { Td: Smagar massif; Uz: Bukhara, Samarkand, } \\
\text { Syrdaryinsk regions }\end{array}$ \\
\hline 143 & Protracheoniscus marginatus (Uljanin, 1875) & Ua: Crimea \\
\hline
\end{tabular}




\begin{tabular}{|c|c|c|}
\hline 144 & Protracheoniscus nogaicus Demianowitz,1931 & Md; Ru: Rostov region \\
\hline 145 & Protracheoniscus orientalis (Uljanin, 1875) & $\begin{array}{l}\text { Az: Baku, Mardakert; Bl: Berezinsky reserve; } \\
\text { Kz: Mangyshlak; Tu: Bugdaily; Ru: Moscow, } \\
\text { Orenburg, Primorie regions; Ua: Kremenetz, } \\
\text { Odessa regions, Uz: Nukus; Zakaspyisk region }\end{array}$ \\
\hline 146 & Protracheoniscus panphilovi Borutzky, 1959 & $\mathrm{Kg}$ \\
\hline 147 & Protracheoniscus politus (C. Koch, 1841) & Ru: Moscow region \\
\hline 148 & $\begin{array}{l}\text { Protracheoniscus scythicus Demianowicz, } \\
1932\end{array}$ & Md \\
\hline 149 & Protracheoniscus steinbergi Borutzky, 1961 & TU: SW part \\
\hline 150 & $\begin{array}{l}\text { Protracheoniscus taschkentensis Verhoeff, } \\
1930\end{array}$ & Uz: Tashkent; Td \\
\hline 151 & Protracheoniscus tashausicus Borutzky, 1976 & Tu; Ru: Rostov region \\
\hline 152 & Protracheoniscus topczievi Borutzky, 1975 & $\begin{array}{l}\text { Ru: Krasnodar, Rostov regions; Ua: Zaporozhye } \\
\text { region }\end{array}$ \\
\hline 153 & $\begin{array}{l}\text { Protracheoniscus tuberculatus (Borutzky, } \\
\text { 1945) }\end{array}$ & $\mathrm{Tu}$ \\
\hline 154 & $\begin{array}{l}\text { Protracheoniscus turcomanicus Borutzky, } \\
1945\end{array}$ & $\mathrm{Tu}$ \\
\hline 155 & Protracheoniscus tzvetkovi Borutzky, 1975 & Kz: Alma-Ata, Uzun-Agach; Ru: Moscow region? \\
\hline 156 & Protracheoniscus uljanini Borutzky, 1953 & $\mathrm{Td}$ \\
\hline 157 & Protracheoniscus verhoeffi Strouhal, 1929 & Ge: Tbilisi \\
\hline 158 & $\begin{array}{l}\text { Protracheoniscus zenkevitschi (Borutzky, } \\
\text { 1945) }\end{array}$ & $\mathrm{Tu}$ \\
\hline 159 & Psachonethes czerkessicus Borutzky, 1969 & Ru: Krasnodar region \\
\hline 160 & $\begin{array}{l}\text { Pseudobuddelundiella hostensis Borutzky, } \\
1967\end{array}$ & Ru: Krasnodar region \\
\hline 161 & $\begin{array}{l}\text { Pseudobuddelundiella ljovuschkini Borutzky, } \\
1967\end{array}$ & Ru: Krasnodar region \\
\hline 162 & Schizidium davidi (Dollfus, 1887) & Az: Divichi \\
\hline 163 & Schizidium golovatchi Schmalfuss, 1988 & Ar: Shikalyukh; Az: Baku; Ge: Batumi \\
\hline 164 & Schizidium reinoehli Schmalfuss, 1988 & Ru: Rostov region \\
\hline 165 & Tadzhikoniscus coecus Borutzky, 1976 & $\mathrm{Td}$ \\
\hline 166 & Tauroligidium stygium Borutzky, 1950 & Ua: Crimea \\
\hline 167 & Tauronethes lebedinskyi Borutzky, 1949 & Ua: Crimea \\
\hline 168 & Titanethes albus (C. Koch, 1841) & Ua: Crimea \\
\hline 169 & $\begin{array}{l}\text { Trachelipus azerbaidzhanus Schmalfuss, } \\
1986\end{array}$ & Az: E part \\
\hline 170 & Trachelipus caucasius (Verhoeff, 1918) & Ab: Gagry; Ru: Krasnodar region \\
\hline 171 & Trachelipus diffcilis Radu, 1950 & Bl: Belovezha, Berezinsky reserves; Ua: S Crimea \\
\hline 172 & Trachelipus ensiculorum Verhoeff, 1949 & Ar: Yerevan \\
\hline 173 & Trachelipus gagriensis (Verhoeff, 1918) & Ab: Gagry \\
\hline 174 & Trachelipus kervillei (Arcangeli, 1938) & Ru: Rostov region \\
\hline 175 & Trachelipus lignaui (Verhoeff, 1918) & Ab: Gagry; Ru: Rostov region \\
\hline 176 & Trachelipus longipennis Budde-Lund, 1885 & Ab; Ua: S Crimea \\
\hline 177 & Trachelipus lutschnikii (Verhoeff, 1933) & Ru: Krasnodar region \\
\hline
\end{tabular}




\begin{tabular}{l|l|l}
\hline 178 & Trachelipus rathkii Brandt, 1833 & $\begin{array}{l}\text { Ab: Sukhum; Az: Airidja; Ar: Chaldyr; Ge: } \\
\text { Kutaisi; Bl: Belovezha pusha; Lt: Vilnius; Md; } \\
\text { Ru: Belgorod, Kursk, Tver, Maryi-El, Kaluga, } \\
\text { Mordovia, Moscow, Penza, Rostov, Leningrad, } \\
\text { Saratov, Tula regions; Ua: Crimea, Kiev }\end{array}$ \\
\hline 179 & Trachelipus razzautii (Arcangeli, 1913) & Ru: Krasnodar region \\
\hline 180 & Trachelipus sarculatus (Budde-Lund, 1896) & Ua: Crimea \\
\hline 181 & Trachelipus trachealis Budde-Lund, 1885 & Md \\
\hline 182 & Trichoniscus aphonicus Borutzky, 1977 & Ab \\
\hline 183 & Trichoniscus gudauticus Borutzky, 1977 & Ab \\
\hline 184 & Trichoniscus pusillus Brandt, 1833 & Ua: Crimea, Kiev \\
\hline 185 & Trichoniscus pygmaeus Sars, 1898 & Ru: Krasnodar region \\
\hline 186 & $\begin{array}{l}\text { Turanoniscus anacanthotermitis Borutzky, } \\
\text { 1969 }\end{array}$ & Uz: Tashkent \\
\hline 187 & Tylos granuliferus Budde-Lund, 1885 & Ru: Primorie region, S Kuril Islands \\
\hline 188 & Tylos ponticus Grebnicki, 1874 & Ua: Crimea, Odessa \\
\hline 189 & Typhloligidium coecum (Carl, 1904) & Ua: Crimea \\
\hline 190 & Typhloligidium karabijajlae Borutzky, 1962 & Ua: Crimea \\
\hline
\end{tabular}

